\title{
Lattice QCD Constraints on the Parton Distribution Functions of ${ }^{3} \mathrm{He}$
}

\author{
William Detmold, ${ }^{1}$ Marc Illa $\odot,{ }^{2}$ David J. Murphy, ${ }^{1}$ Patrick Oare $\odot,{ }^{1}$ Kostas Orginos,,${ }^{3,4}$ \\ Phiala E. Shanahan $\odot,{ }^{1}$ Michael L. Wagman, ${ }^{5}$ and Frank Winter $\oplus^{4}$
}

(NPLQCD Collaboration)

\author{
${ }^{1}$ Center for Theoretical Physics, Massachusetts Institute of Technology, Cambridge, Massachusetts 02139, USA \\ ${ }^{2}$ Departament de Física Quàntica i Astrofísica and Institut de Ciències del Cosmos, \\ Universitat de Barcelona, Martí i Franquès 1, 08028 Barcelona, Spain \\ ${ }^{3}$ Department of Physics, College of William and Mary, Williamsburg, Virginia 23187-8795, USA \\ ${ }^{4}$ Jefferson Laboratory, 12000 Jefferson Avenue, Newport News, Virginia 23606, USA \\ ${ }^{5}$ Fermi National Accelerator Laboratory, Batavia, Illinois 60510, USA
}

(Received 22 September 2020; accepted 19 April 2021; published 17 May 2021)

\begin{abstract}
The fraction of the longitudinal momentum of ${ }^{3} \mathrm{He}$ that is carried by the isovector combination of $u$ and $d$ quarks is determined using lattice QCD for the first time. The ratio of this combination to that in the constituent nucleons is found to be consistent with unity at the few-percent level from calculations with quark masses corresponding to $m_{\pi} \sim 800 \mathrm{MeV}$. With a naive extrapolation to the physical quark masses, this constraint is consistent with, and more precise than, determinations from global nuclear parton distribution function fits through the nNNPDF framework. It is thus concretely demonstrated that lattice QCD calculations of light nuclei have imminent potential to enable more precise determinations of the $u$ and $d$ parton distributions in light nuclei and to reveal the QCD origins of the EMC effect.
\end{abstract}

DOI: 10.1103/PhysRevLett.126.202001

A central pillar of our understanding of the internal structure of strongly interacting hadronic and nuclear systems is knowledge of their partonic structure as accessed in deep-inelastic scattering experiments and other hard processes. Since the 1960s, such experiments have revealed the longitudinal momentum distributions of quarks and gluons in a fast moving proton, known collectively as parton distribution functions (PDFs). The simplest PDFs, $q(x, \mu)$ [and $g(x, \mu)$ ], describe the probability of a quark of flavor $q$ (or gluon $g$ ) carrying a fraction $x$ of the longitudinal momentum of the struck proton at a renormalization scale $\mu$. In 1983, the European Muon Collaboration (EMC) [1] observed that the partonic structure of nuclei differs substantially from that of the constituent protons and neutrons, a landmark in the development of nuclear physics [2-6]. Since the deepinelastic scattering processes observed in the EMC experiments were at very high energy and the binding energy of a nucleus is small in comparison to its mass, the appearance and size of the EMC effect was surprising

Published by the American Physical Society under the terms of the Creative Commons Attribution 4.0 International license. Further distribution of this work must maintain attribution to the author(s) and the published article's title, journal citation, and DOI. Funded by SCOAP . at the time. Interest in the EMC effect has been rekindled by recent data from SLAC and Jefferson Lab [7-11] on EMC ratios for light nuclei. Not only have these data provided precise determinations of the EMC effect for nuclei with small atomic number $A$, but they have revealed a correlation between the strength of the EMC effect and so called "short range correlations" $[12,13]$.

In addition to experimental investigations, theoretical calculations of the partonic structure of hadrons and nuclei from the standard model can have important impacts on our understanding of the structure of matter. For example, standard model calculations of nuclear partonic structure would reveal the QCD origin of the EMC effect as well as aid in the flavor separation of proton PDFs. Parton distributions are inherently rooted in the strong interaction dynamics of QCD and cannot be determined using perturbative methods. Since the seminal works of Refs. [14,15], lattice quantum chromodynamics (LQCD) calculations have addressed the simplest aspects of the parton distributions of the proton, notably determining the first few Mellin moments of the unpolarized, polarized, and transversity quark distributions [16], as well as their gluonic analogs [17-21]. Recently, efforts have been made to extend these studies to the full $x$ dependence of the proton PDFs [16,22-28]. More complicated extensions of partonic structure, such as generalized parton distribution functions 
and transverse-momentum-dependent parton distribution functions of the proton, have also been studied using LQCD [29-34].

In this Letter, the partonic structure of light nuclei is studied in LQCD for the first time through an investigation of the isovector quark momentum fractions (the first moments of the corresponding isovector PDFs) of the proton, diproton, and ${ }^{3} \mathrm{He}$. At the heavier-than-physical quark masses used in this LQCD study, percent-level nuclear effects are resolved in the momentum fraction of ${ }^{3} \mathrm{He}$. While a rigorous chiral extrapolation cannot be undertaken using these results, a naive extrapolation to the physical quark masses can be performed, and the resulting constraint on the isovector momentum fraction used as an additional input into the nNNPDF2.0 [35] global nuclear PDF analysis framework. Since the isovector combination of nuclear PDFs is poorly determined from experiment, even with significant estimated uncertainties from the chiral extrapolation, this LQCD constraint reduces the uncertainties on the ${ }^{3} \mathrm{He}$ PDFs and thereby demonstrates a concrete synergy between experiment and LQCD in the quest to improve knowledge of nuclear structure.

LQCD methodology.-The existence of strong interactions between quarks and gluons necessitates the use of LQCD for calculations of the partonic structure of nuclei. The calculations presented here are performed using a single ensemble of gauge-field configurations generated with a Lüscher-Weisz gauge action [36] with $N_{f}=3$ degenerate light-quark flavors with the clover-improved Wilson fermion action [37] and quark masses tuned to produce a pion mass of $m_{\pi}=806 \mathrm{MeV}$. The lattice geometry is $L^{3} \times T=32^{3} \times 48$, and the lattice spacing is determined to be $a \sim 0.145 \mathrm{fm}$ from $\Upsilon$ spectroscopy [38]. This ensemble and two others with different spacetime volumes have previously been used to study the spectrum [38,39] and properties [40-50] of light nuclei up to atomic number $A=4$. The multivolume spectroscopy studies show that the $p p$ and ${ }^{3} \mathrm{He}$ states that are investigated here are bound systems with infinite volume energies below threshold. Consequently, matrix elements in these states are expected to receive only exponentially small finite volume effects, $\mathcal{O}\left(e^{-\kappa L}, e^{-m_{\pi} L}\right)$, that will be neglected in this work [51-57].

The Mellin moments of the unpolarized isovector quark PDFs, $q_{3}^{(h)}(x, \mu)=u^{(h)}(x, \mu)-d^{(h)}(x, \mu)$, in a hadronic or nuclear state $h$, defined as $\left\langle x^{n}\right\rangle_{u-d}^{(h)}(\mu) \equiv$ $\int_{-1}^{1} d x x^{n} q_{3}^{(h)}(x, \mu)$, are determined from matrix elements of twist-two operators as

$$
\begin{aligned}
\left\langle h\left|\mathcal{O}_{\mu_{0} \ldots \mu_{n}}\right| h\right\rangle & \equiv\left\langle h\left|\bar{q} \tau_{3} \gamma_{\left\{\mu_{0}\right.}\left(i \stackrel{\leftrightarrow}{D}_{\mu_{1}}\right) \ldots\left(i \stackrel{\leftrightarrow}{D}_{\left.\mu_{n}\right\}}\right) q\right| h\right\rangle \\
& =\left\langle x^{n}\right\rangle_{u-d}^{(h)}(\mu) p_{\left\{\mu_{0}\right.} \ldots p_{\left.\mu_{n}\right\}},
\end{aligned}
$$

where $p$ is the momentum of the state $h, \tau_{3}$ is a Pauli matrix in flavor space, $\stackrel{\leftrightarrow}{D}_{\mu}=\left(\vec{D}_{\mu}-\overleftarrow{D}_{\mu}\right) / 2$ where $D_{\mu}$ is the gauge covariant derivative, and $\{\ldots\}$ indicates symmetrization and trace subtraction of the enclosed indices. The above operators are constructed to transform irreducibly under the Lorentz group, but the hypercubic spacetime lattice used in the LQCD calculations reduces these symmetries, in general inducing mixing between operators of different Lorentz spin. In particular, the two-index operators that determine the isovector quark momentum fraction $\langle x\rangle_{u-d}^{(h)}$ subduce to operators in two different irreducible representations of the hypercubic group. In this work, matrix elements of an Euclidean operator in the $\tau_{1}^{(3)}$ representation [58] are computed, namely

$\mathcal{T}=\frac{1}{\sqrt{2}}\left(\mathcal{T}_{33}-\mathcal{T}_{44}\right), \quad$ with $\quad \mathcal{T}_{\mu \nu}=\bar{q} \tau_{3} \gamma_{\{\mu} \stackrel{\leftrightarrow}{D}_{\nu\}} q$,

where $\gamma_{\nu}$ is also Euclidean. With a lattice regulator, this operator is discretized as a covariant finite difference whose form is given in the Supplemental Material [59]. For both spinzero and spin-half systems, spin-averaged in the latter case, matrix elements in states with zero three-momentum determine the momentum fraction as $\langle h|\mathcal{T}| h\rangle=\langle x\rangle_{u-d}^{(h)} M_{h} / \sqrt{2}$.

The renormalized operator in the modified minimal subtraction scheme $(\overline{\mathrm{MS}})$ is related to the bare lattice operator in Eq. (2) as

$\mathcal{T}^{(\overline{\mathrm{MS}})}(\mu)=\mathcal{R}^{\overline{\mathrm{MS}} / \mathrm{RI}^{\prime} \mathrm{MOM}}\left(\mu, \mu_{0}\right) \mathcal{Z}^{\mathrm{RI} \mathrm{MOM}}\left(\mu_{0}, a\right) \mathcal{T}(a)$,

where the renormalization coefficient $\left.\mathcal{Z}^{\mathrm{RI}} \mathrm{ROM}^{\prime} \mathrm{MO}, a\right)$ is defined nonperturbatively in a regularization-independent momentum-subtraction scheme [60] at a scale $\mu_{0}$ and then matched to $\overline{\mathrm{MS}}$ through the three-loop perturbative coefficient $\mathcal{R}^{\overline{\mathrm{MS}} / \mathrm{RI} \mathbf{R O M}^{\prime}}\left(\mu, \mu_{0}\right)$ [61,62], as detailed in the Supplemental Material [59]. For $\mu=2 \mathrm{GeV}$, $\mathcal{R}^{\overline{\mathrm{MS}} / \mathrm{RI} \mathrm{MOM}}\left(\mu, \mu_{0}\right) \mathcal{Z}^{\mathrm{RI}^{\prime} \mathrm{MOM}}\left(\mu_{0}, a\right)=0.89(4)$.

The techniques needed to compute matrix elements of this operator are simple generalizations of those used for calculations of isovector matrix elements of quark currents using the compound-propagator background-field method [63] introduced in Ref. [46] and further detailed in Refs. [47,48,64] and the Supplemental Material [59]. Quark propagators and $\mathcal{T}$-compound propagators are computed from an average of $N_{\text {src }}=24$ source points randomly distributed on $N_{\mathrm{cfg}}=2290$ gauge-field configurations for $N_{B}=5$ different background-field strengths. These compound propagators are then used to construct baryon two-point correlation functions,

$$
G_{h}(t ; \lambda)=\sum_{\mathbf{x}}\left\langle 0\left|\chi_{h}(\mathbf{x}, t) \chi_{h}^{\dagger}(0)\right| 0\right\rangle_{\lambda},
$$

where $\lambda$ is the $\mathcal{T}$-background-field strength, $\chi_{h}$ is an interpolating field for states with the quantum numbers of the hadron or nucleus $h$, and spinor indices on the 
interpolating operators are suppressed. Correlation functions are constructed from Gaussian-smeared source interpolating operators [65], while the sink interpolating operators are either smeared or pointlike, and the multibaryon contractions are performed using the techniques of Ref. [66]. This quantity contains responses to the field up to $\mathcal{O}\left(\lambda^{N_{Q}}\right)$, with $N_{Q}$ being the number of valence quarks in the state. The linear response of this background-field twopoint function, $\left.G_{h}(t ; \lambda)\right|_{\mathcal{O}(\lambda)}$, is determined by the matrix element of $\mathcal{T}$. This term can be extracted exactly from the computed set of fixed-order background-field correlation functions with $N_{Q}$ field strengths [46,47].

Combining the linear response of the two-point correlation function with the zero-field correlation function, it is straightforward to show that the ratio

$$
\mathcal{R}_{h}(t)=\frac{\left.G_{h}(t+a ; \lambda)\right|_{\mathcal{O}(\lambda)}}{a G_{h}(t+a ; 0)}-\frac{\left.G_{h}(t ; \lambda)\right|_{\mathcal{O}(\lambda)}}{a G_{h}(t ; 0)}
$$

is related to matrix elements of $\mathcal{T}$ through the spectral representation of each term in Eq. (5), in particular asymptoting as

$$
\mathcal{R}_{h}(t) \stackrel{t \rightarrow \infty}{\rightarrow}\langle h|\mathcal{T}| h\rangle,
$$

with exponentially vanishing contamination at early times that involves excited-state overlap factors and transition matrix elements.

Ground-state matrix elements are extracted from $\mathcal{R}_{h}(t)$, and systematic fitting uncertainties are estimated using a procedure for sampling from all possible fit ranges and models analogous to the procedure described for two-point correlation functions in Ref. [67]. In summary, in analyzing $\mathcal{R}_{h}(t)$ to extract the momentum fractions, the full $t$ dependence that results from the spectral decomposition of each term in Eq. (5) is fit, and combined fits to two- and three-point correlation functions are used to constrain the relevant energies, overlap factors, and matrix elements. All possible choices of fit ranges and up to four states contributing to the spectral decompositions are considered using a model selection process described in the Supplemental Material [59]. A weighted average over fits from all acceptable fit ranges is used to define ground-state energies, including systematic uncertainties from fit range and model variation. Results are shown in Fig. 1 for the proton, diproton, and ${ }^{3} \mathrm{He}$.

Results and discussion.-The extracted values of the isovector quark momentum fractions for $p, p p,{ }^{3} \mathrm{He}$ at quark masses corresponding to $m_{\pi}=m_{K}=806 \mathrm{MeV}$ are shown in Table I and displayed graphically in Fig. 2. The uncertainties are separated into those from the LQCD calculation of the bare matrix elements and the (larger) uncertainty from the renormalization and matching to the $\overline{\mathrm{MS}}$ scheme. The proton isovector momentum fraction is

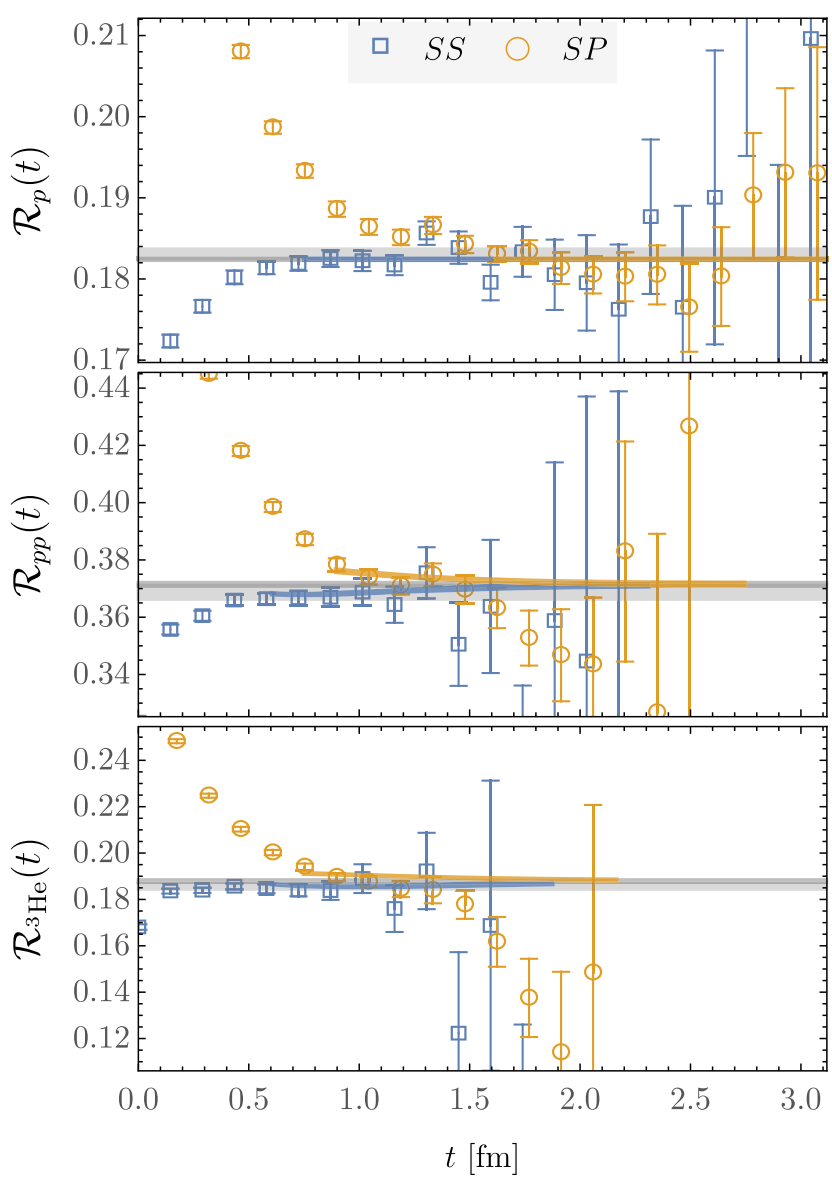

FIG. 1. The effective matrix element, Eq. (5), associated with the isovector quark momentum fractions of the proton, $p p$ and ${ }^{3}$ He. Blue (orange) points, labeled SS (SP), show results for interpolating operators with smeared sources and smeared (pointlike) sinks. For each effective matrix element, points are shown for $t \leq t_{\max }$, where $t_{\max }$ is the minimum $t$ where the signal-tonoise ratio of $\left.G_{h}(t+a ; \lambda)\right|_{\mathcal{O}(\lambda)}$ is less than 0.5. Colored bands show the highest weight fit to the combined dataset, and the shaded gray bands show the weighted average of all accepted fits and the total statistical plus fitting systematic uncertainties.

consistent with other LQCD extractions at similar values of the quark masses [68] given the different renormalization procedures and lattice spacings. The $p p$ and ${ }^{3} \mathrm{He}$

TABLE I. The isovector quark momentum fractions in $p, p p$, and ${ }^{3} \mathrm{He}$ calculated at $m_{\pi}=806 \mathrm{MeV}$ in $\overline{\mathrm{MS}}$ scheme at $\mu=2 \mathrm{GeV}$. The first uncertainty combines LQCD statistical and systematic uncertainties, and the second uncertainty is from operator renormalization. The correlated ratios of the isovector momentum fraction in nuclei to those in the constituent nucleons, in which the renormalization constants and their uncertainties cancel, are also given.

\begin{tabular}{lccc}
\hline \hline & $p$ & $p p$ & ${ }^{3} \mathrm{He}$ \\
\hline$\langle x\rangle_{u-d}^{(h)}$ & $0.191(1)(9)$ & $0.194(2)(9)$ & $0.066(1)(3)$ \\
$(A / Z-N)\langle x\rangle_{u-d}^{(h)} /\langle x\rangle_{u-d}^{(p)}$ & $\cdots$ & $1.007(14)$ & $1.028(15)$ \\
\hline \hline
\end{tabular}




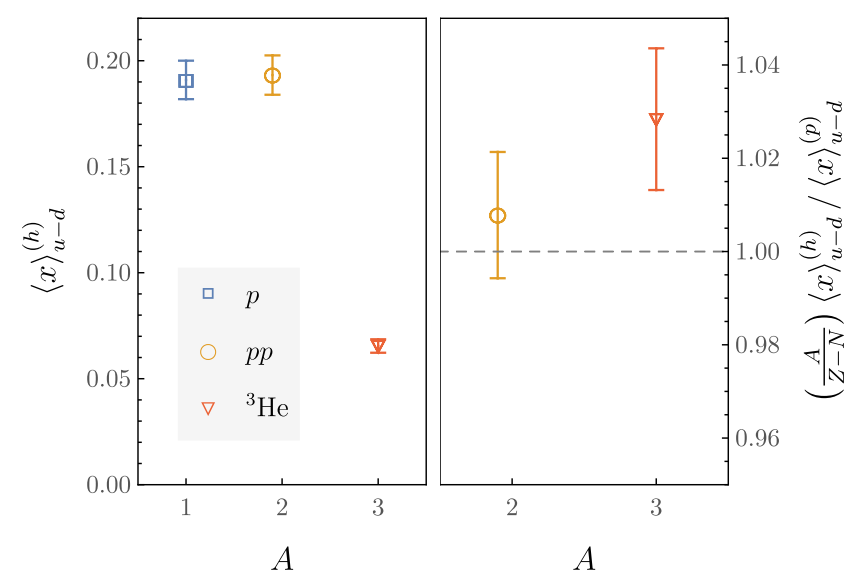

FIG. 2. Left: Renormalized isovector momentum fractions for $h \in\left\{p, p p,{ }^{3} \mathrm{He}\right\}$ at a scale of $\mu=2 \mathrm{GeV}$. Right: Ratios of the isovector nuclear momentum fractions to that of the constituent nucleons.

momentum fractions are determined with $\mathcal{O}(5 \%)$ uncertainties and are found to be approximately consistent with those of the constituent nucleons. The ratios of the nuclear momentum fractions to that of the proton are independent of operator renormalization to $\mathcal{O}\left(\alpha_{s}\right)$ and are determined at few-percent precision even for ${ }^{3} \mathrm{He}$.

In Refs. [69-71], nuclear effective field theory (EFT) was used to study nuclear effects in PDF moments. In particular, it was shown that the leading source of such effects is the two-nucleon correlations that couple to the twist-two operators defining the PDF moments. In terms of the parameters defined in that work, nuclear effects in the isovector momentum fraction are encapsulated in the low energy constant (LEC) $\alpha_{3,2}$ and nuclear factor $\mathcal{G}_{3}\left({ }^{3} \mathrm{He}\right)$; their product is bounded as $\alpha_{3,2} \mathcal{G}_{3}\left({ }^{3} \mathrm{He}\right)=0.0018(14)$ at $\mu=2 \mathrm{GeV}$ from the numerical calculations presented here (see the Supplemental Material [59] for details). While the quark momentum fractions themselves have nonanalytic dependence on the quark masses [72-74], from powercounting in chiral EFT it is natural to expect that the higherorder mass dependence in the product $\alpha_{3,2} \mathcal{G}_{3}\left({ }^{3} \mathrm{He}\right)$ is suppressed relative to the constant part; how well that expectation persists in studies with such an unphysically large pion mass remains to be tested quantitatively. However, in the only cases for which analogous LECs have been investigated at the quark masses used here, as well as at lighter masses (the analogous two-body contribution in the $n p \rightarrow d \gamma$ [43] process, as well as the LECs describing the leading-order strong two-nucleon interactions [75,76]), there was little pion-mass dependence in the relevant LECs. Adopting an assumption of relative mass independence based on this observation allows an extrapolation of $\alpha_{3,2} \mathcal{G}_{3}\left({ }^{3} \mathrm{He}\right)$ to the physical quark masses: a naive estimate is given by taking the central value determined at $m_{\pi}=806 \mathrm{MeV}$ and adding in quadrature with the statistical and systematic uncertainties an additional uncertainty of $100 \%$ the value of the LEC itself to account for possible quark-mass dependence as well as the effects of the nonzero lattice spacing and finite volume. (As detailed in the Supplemental Material [59], this uncertainty is estimated based on the mass dependence seen in the other two-body LECs.) To account for the level of uncertainty in this estimate, extrapolations are also undertaken with 50\% and $200 \%$ of the central value of the LEC added as an additional systematic uncertainty. These extrapolated values can be combined with the physical value of the nucleon momentum fraction, $\langle x\rangle_{u-d}^{(p)}=0.160(7)$ at $\mu=2 \mathrm{GeV}$ from the nNNPDF2.0 analysis [35], to determine the isovector momentum fraction ratio $3\langle x\rangle_{u-d}^{(3 \mathrm{He})} /\left.\langle x\rangle_{u-d}^{(p)}\right|_{\mathrm{LQCD}}=$ $1.033(38)$ at the physical quark masses [or alternatively $1.033(25)$ or $1.033(69)$ with $50 \%$ or $200 \%$ estimated systematics as discussed above].

It is interesting to compare the LQCD results for the momentum fractions and their ratios to phenomenology. In particular, the isovector momentum fractions determined here provide valuable information that is complementary to experimental constraints on the nuclear modification of PDFs; almost all information on the nuclear modification of partonic structure has been obtained for the ratio of isoscalar-corrected $F_{2}$ structure functions of nuclei to that of the deuteron $[3,5,6]$. Additional constraints are especially valuable in the context of the intriguing question as to whether there is flavor dependence to the EMC effect. Such flavor dependence has been conjectured in models of QCD [77-82] and in EFT [69-71] and is included in recent datadriven analyses of experimental results $[83,84]$ and provides a potential explanation of the $\mathrm{NuTeV}$ anomaly in $\sin ^{2} \theta_{W}[85]$.

Figure 3 shows the constraint on the isovector momentum fraction ratio for ${ }^{3} \mathrm{He}$ obtained from the results presented here compared to the constraints on the isovector and isoscalar momentum fraction ratios from the recent nNNPDF2.0 [35] global nuclear PDF fits. The nNNPDF2.0 ellipse is generated by combining the Monte Carlo replica sets for the bound proton PDFs in ${ }^{4} \mathrm{He}$ appropriately to form the PDFs of ${ }^{3} \mathrm{He}$ (under the assumption that the nuclear effects vary slowly with $A$ ). In this way, correlations between the ${ }^{3} \mathrm{He}$ and proton PDFs are accounted for. For the isovector combination, the $68 \%$ confidence interval is $3\langle x\rangle_{u-d}^{\left({ }^{3} \mathrm{He}\right)} /\left.\langle x\rangle_{u-d}^{(p)}\right|_{\mathrm{nNNPDF} 2.0}=1.007(63)$. In the nNNPDF approach, it is also straightforward to impose the LQCD constraint on the nuclear PDFs by reweighting the Monte Carlo replicas as discussed in Ref. [86]; the combined confidence region is shown in Fig. 3. The $68 \%$ confidence interval reduces to $3\langle x\rangle_{u-d}^{\left({ }^{3} \mathrm{He}\right)} /\left.\langle x\rangle_{u-d}^{(p)}\right|_{\mathrm{nNNPDF} 20+\mathrm{LQCD}}=1.024(32) \quad$ or alternatively $1.029(23)$ or $1.017(43)$ with the more aggressive and less aggressive estimates of the uncertainty in the chiral extrapolation discussed above]. As can be seen from Fig. 3, 


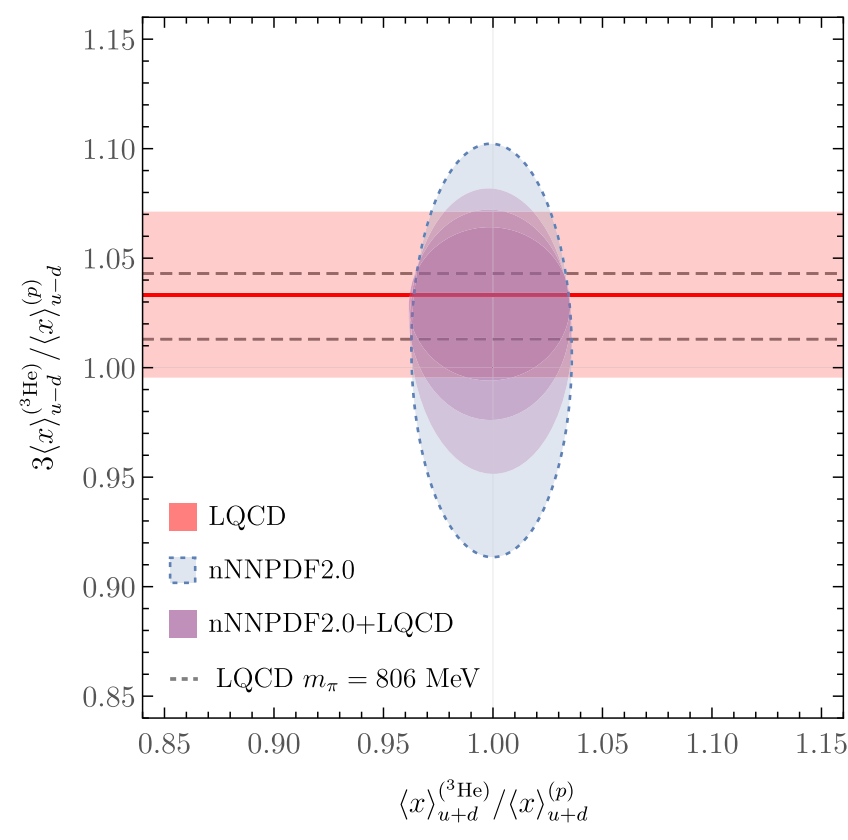

FIG. 3. The ratio of the isovector momentum fractions of ${ }^{3} \mathrm{He}$ and $p$ determined in this work compared to constraints on the isovector and isoscalar momentum fraction ratios from the nNNPDF2.0 [35] global analysis before and after the LQCD constraint is imposed. Both axes are normalized to unity in the absence of nuclear effects. The red band, and middle purple ellipse, correspond to LQCD results taking $100 \%$ of the value of the LEC as an additional systematic uncertainty to account for its unconstrained mass dependence, as discussed in the text. The inner and outer purple ellipses show the results of taking 50\% and $200 \%$ of the LEC as the additional systematic uncertainty before incorporating the LQCD constraint into the global fit. The LQCD constraint on the isovector ratio at $m_{\pi}=806 \mathrm{MeV}$ is also displayed. In all cases, $68 \%$ confidence intervals are shown.

even with a conservative estimate of uncertainties resulting from the larger-than-physical pion mass of the calculations, LQCD calculations such as those presented here, as well as new experimental constraints $[87,88]$, can significantly improve our knowledge of the flavor dependence of nuclear PDFs.

Summary.-In this work, the isovector momentum fractions of the proton, diproton, and ${ }^{3} \mathrm{He}$ systems have been determined using LQCD, complementing a previous study of the gluon momentum fraction on the same ensemble [49]. These calculations were performed at a single set of unphysical SU(3)-symmetric values for the quark masses corresponding to $m_{\pi}=806 \mathrm{MeV}$ and in a single lattice volume and at a single lattice spacing. Bearing these caveats in mind, the isovector nuclear momentum fractions were calculated precisely and found to be similar to that of the proton. In particular, the ratios $\langle x\rangle_{u-d}^{(p p)} /\langle x\rangle_{u-d}^{(p)}=$ $1.010(14)$ and $3\langle x\rangle_{u-d}^{\left({ }^{3} \mathrm{He}\right)} /\langle x\rangle_{u-d}^{(p)}=1.029(15)$ were determined and nuclear EFT arguments were used to connect the ${ }^{3} \mathrm{He}$ result to global analyses of nuclear PDFs, providing constraints on the flavor decomposition of nuclear PDFs that are complementary to those obtained from experiment.

While still in its early stages, this work emphasizes the utility of LQCD in constraining less well-measured aspects of partonic structure in an analogous way to how LQCD inputs have been used to constrain the proton transversity PDFs [89]. Future calculations at the physical quark masses will consider higher moments of nuclear PDFs (or even directly study their $x$ dependence) for a wider range of nuclei and provide a complete flavor decomposition. Calculations will also quantitatively address the full set of systematic uncertainties.

We thank Juan Rojo for comments and substantial help with the comparison to the nNNPDF2.0 global fits, and Silas Beane, Jiunn-Wei Chen, Zohreh Davoudi, Assumpta Parreño, Martin Savage, and Brian Tiburzi for insightful discussions. This research used resources of the Oak Ridge Leadership Computing Facility at the Oak Ridge National Laboratory, which is supported by the Office of Science of the U.S. Department of Energy under Contract No. DEAC05-00OR22725, as well as facilities of the USQCD Collaboration, which are funded by the Office of Science of the U.S. Department of Energy. This research used resources of the National Energy Research Scientific Computing Center (NERSC), a U.S. Department of Energy Office of Science User Facility operated under Contract No. DEAC02-05CH11231. The CHROMA [90], QLUA [91], QUDA [92,93], QDP-JIT [94], and QPHIX [95] software libraries were used in data production and analysis. W. D., D. J. M., and P. E. S. acknowledge support from the U.S. DOE Grant No. DE-SC0011090. W. D. is also supported within the framework of the TMD Topical Collaboration of the U.S. DOE Office of Nuclear Physics and by the SciDAC4 Award No. DE-SC0018121. P. E. S. is additionally supported by the National Science Foundation under CAREER Grant No. 1841699 and under EAGER Grant No. 2035015, by the U.S. DOE Early Career Award No. DE-SC0021006, by an NEC research award, and by the Carl G and Shirley Sontheimer Research Fund. M. I. is supported by the Universitat de Barcelona through the scholarship APIF by the Spanish Ministerio de Economía y Competitividad (MINECO) under the Project No. MDM-2014-0369 of ICCUB (Unidad de Excelencia "María de Maeztu") and with additional European FEDER funds under the Contract No. FIS2017-87534-P. K. O. was supported in part by U.S. DOE Grant No. DE-FG02-04ER41302 and in part by the Jefferson Science Associates, LLC under U.S. DOE Contract No. DE-AC05-06OR23177. This manuscript has been authored by Fermi Research Alliance, LLC under Contract No. DE-AC02-07CH11359 with the U.S. Department of Energy, Office of Science, Office of High Energy Physics. The authors thank Robert Edwards, Bálint Joó, and members of the NPLQCD collaboration for generating and allowing access to the ensembles used in this study, as well as for helpful discussions.erdf 
[1] J. J. Aubert et al. (European Muon Collaboration), Phys. Lett. B 123, 275 (1983).

[2] M. Arneodo, Phys. Rep. 240, 301 (1994).

[3] D. F. Geesaman, K. Saito, and A. W. Thomas, Annu. Rev. Nucl. Part. Sci. 45, 337 (1995).

[4] G. Piller and W. Weise, Phys. Rep. 330, 1 (2000).

[5] P. R. Norton, Rept. Prog. Phys. 66, 1253 (2003).

[6] O. Hen, D. W. Higinbotham, G. A. Miller, E. Piasetzky, and L. B. Weinstein, Int. J. Mod. Phys. E 22, 1330017 (2013).

[7] N. Fomin, J. Arrington, R. Asaturyan, F. Benmokhtar, W. Boeglin, P. Bosted, A. Bruell, M. H. S. Bukhari, M.E. Christy, E. Chudakov et al., Phys. Rev. Lett. 108, 092502 (2012).

[8] O. Hen, E. Piasetzky, and L. B. Weinstein, Phys. Rev. C 85, 047301 (2012).

[9] L. L. Frankfurt, M. I. Strikman, D. B. Day, and M. Sargsian, Phys. Rev. C 48, 2451 (1993).

[10] K. S. Egiyan et al. (CLAS Collaboration), Phys. Rev. C 68, 014313 (2003).

[11] K. S. Egiyan et al. (CLAS Collaboration), Phys. Rev. Lett. 96, 082501 (2006).

[12] L. B. Weinstein, E. Piasetzky, D. W. Higinbotham, J. Gomez, O. Hen, and R. Shneor, Phys. Rev. Lett. 106, 052301 (2011).

[13] O. Hen, E. Piasetzky, and L. B. Weinstein, Phys. Rev. C 85, 047301 (2012).

[14] G. Martinelli and C. T. Sachrajda, Nucl. Phys. B306, 865 (1988).

[15] G. Martinelli and C. T. Sachrajda, Nucl. Phys. B316, 355 (1989).

[16] H.-W. Lin et al., Prog. Part. Nucl. Phys. 100, 107 (2018).

[17] R. Horsley, R. Millo, Y. Nakamura, H. Perlt, D. Pleiter, P. E. L. Rakow, G. Schierholz, A. Schiller, F. Winter, and J. M. Zanotti (UKQCD and QCDSF Collaborations), Phys. Lett. B 714, 312 (2012).

[18] C. Alexandrou, M. Constantinou, K. Hadjiyiannakou, K. Jansen, H. Panagopoulos, and C. Wiese, Phys. Rev. D 96, 054503 (2017).

[19] Y.-B. Yang, M. Gong, J. Liang, H.-W. Lin, K.-F. Liu, D. Pefkou, and P. Shanahan, Phys. Rev. D 98, 074506 (2018).

[20] P. E. Shanahan and W. Detmold, Phys. Rev. D 99, 014511 (2019).

[21] P. E. Shanahan and W. Detmold, Phys. Rev. Lett. 122, 072003 (2019).

[22] X. Ji, Phys. Rev. Lett. 110, 262002 (2013).

[23] A. Radyushkin, Int. J. Mod. Phys. A 35, 2030002 (2020).

[24] B. Joó, J. Karpie, K. Orginos, A. Radyushkin, D. Richards, and S. Zafeiropoulos, J. High Energy Phys. 12 (2019) 081.

[25] K. Cichy and M. Constantinou, Adv. High Energy Phys. 2019, 1 (2019).

[26] X. Ji, Y.-S. Liu, Y. Liu, J.-H. Zhang, and Y. Zhao, arXiv:2004.03543.

[27] K. Can et al., Phys. Rev. D 102, 114505 (2020).

[28] A. J. Chambers, R. Horsley, Y. Nakamura, H. Perlt, P. E. L. Rakow, G. Schierholz, A. Schiller, K. Somfleth, R. D. Young, and J. M. Zanotti, Phys. Rev. Lett. 118, 242001 (2017).

[29] P. Hagler, Phys. Rep. 490, 49 (2010).

[30] B. Yoon, M. Engelhardt, R. Gupta, T. Bhattacharya, J. R. Green, B. U. Musch, J. W. Negele, A. V. Pochinsky,
A. Schäfer, and S. N. Syritsyn, Phys. Rev. D 96, 094508 (2017).

[31] P. Shanahan, M. Wagman, and Y. Zhao, Phys. Rev. D 102, 014511 (2020).

[32] C. Alexandrou, K. Cichy, M. Constantinou, K. Hadjiyiannakou, K. Jansen, A. Scapellato, and F. Steffens, Phys. Rev. Lett. 125, 262001 (2020).

[33] H.-W. Lin, arXiv:2008.12474.

[34] Q.-A. Zhang et al. (Lattice Parton Collaboration), Phys. Rev. Lett. 125, 192001 (2020).

[35] R. Abdul Khalek, J. J. Ethier, J. Rojo, and G. van Weelden, J. High Energy Phys. 09 (2020) 183.

[36] M. Lüscher and P. Weisz, Commun. Math. Phys. 97, 59 (1985); 98, 433(E) (1985).

[37] B. Sheikholeslami and R. Wohlert, Nucl. Phys. B259, 572 (1985).

[38] S. R. Beane, E. Chang, S. D. Cohen, W. Detmold, H. W. Lin, T. C. Luu, K. Orginos, A. Parreño, M. J. Savage, and A. Walker-Loud (NPLQCD Collaboration), Phys. Rev. D 87, 034506 (2013).

[39] M. L. Wagman, F. Winter, E. Chang, Z. Davoudi, W. Detmold, K. Orginos, M. J. Savage, and P. E. Shanahan, Phys. Rev. D 96, 114510 (2017).

[40] S. R. Beane et al., arXiv:1705.09239.

[41] S. R. Beane et al. (NPLQCD Collaboration), Phys. Rev. C 88, 024003 (2013).

[42] S. R. Beane, E. Chang, S. Cohen, W. Detmold, H. W. Lin, K. Orginos, A. Parreño, M. J. Savage, and B. C. Tiburzi, Phys. Rev. Lett. 113, 252001 (2014).

[43] S. R. Beane, E. Chang, W. Detmold, K. Orginos, A. Parreño, M. J. Savage, and B. C. Tiburzi (NPLQCD Collaboration), Phys. Rev. Lett. 115, 132001 (2015).

[44] E. Chang, W. Detmold, K. Orginos, A. Parreño, M. J. Savage, B. C. Tiburzi, and S. R. Beane (NPLQCD Collaboration), Phys. Rev. D 92, 114502 (2015).

[45] W. Detmold, K. Orginos, A. Parreño, M. J. Savage, B. C. Tiburzi, S. R. Beane, and E. Chang, Phys. Rev. Lett. 116, 112301 (2016).

[46] M. J. Savage, P. E. Shanahan, B. C. Tiburzi, M. L. Wagman, F. Winter, S. R. Beane, E. Chang, Z. Davoudi, W. Detmold, and K. Orginos, Phys. Rev. Lett. 119, 062002 (2017).

[47] B. C. Tiburzi, M. L. Wagman, F. Winter, E. Chang, Z. Davoudi, W. Detmold, K. Orginos, M. J. Savage, and P. E. Shanahan, Phys. Rev. D 96, 054505 (2017).

[48] P. E. Shanahan, B. C. Tiburzi, M. L. Wagman, F. Winter, E. Chang, Z. Davoudi, W. Detmold, K. Orginos, and M. J. Savage, Phys. Rev. Lett. 119, 062003 (2017).

[49] F. Winter, W. Detmold, A. S. Gambhir, K. Orginos, M. J. Savage, P. E. Shanahan, and M. L. Wagman, Phys. Rev. D 96, 094512 (2017).

[50] E. Chang, Z. Davoudi, W. Detmold, A. S. Gambhir, K. Orginos, M. J. Savage, P. E. Shanahan, M. L. Wagman, and F. Winter (NPLQCD Collaboration), Phys. Rev. Lett. 120, 152002 (2018).

[51] M. Lüscher, Commun. Math. Phys. 104, 177 (1986).

[52] M. Lüscher, Commun. Math. Phys. 105, 153 (1986).

[53] M. Lüscher, Nucl. Phys. B354, 531 (1991).

[54] S. Beane, P. Bedaque, A. Parreño, and M. Savage, Phys. Lett. B 585, 106 (2004). 
[55] Z. Davoudi and M. J. Savage, Phys. Rev. D 84, 114502 (2011).

[56] S. König and D. Lee, Phys. Lett. B 779, 9 (2018).

[57] R. A. Briceño, M. T. Hansen, and A. W. Jackura, Phys. Rev. D 100, 114505 (2019).

[58] M. Gockeler, R. Horsley, E.-M. Ilgenfritz, H. Perlt, P. E. L. Rakow, G. Schierholz, and A. Schiller, Phys. Rev. D 54, 5705 (1996).

[59] See Supplemental Material at http://link.aps.org/supplemental/ 10.1103/PhysRevLett.126.202001 for further details of the matrix element extraction, nonperturbative renormalisation, and the extrapolation to the physical quark masses.

[60] G. Martinelli, C. Pittori, C. T. Sachrajda, M. Testa, and A. Vladikas, Nucl. Phys. B445, 81 (1995).

[61] J. Gracey, Nucl. Phys. B667, 242 (2003).

[62] J. Gracey, Nucl. Phys. B662, 247 (2003).

[63] W. Detmold, Phys. Rev. D 71, 054506 (2005).

[64] C. Bouchard, C. C. Chang, T. Kurth, K. Orginos, and A. Walker-Loud, Phys. Rev. D 96, 014504 (2017).

[65] M. Albanese et al. (APE Collaboration), Phys. Lett. B 192, 163 (1987).

[66] W. Detmold and K. Orginos, Phys. Rev. D 87, 114512 (2013).

[67] S. Beane et al., Phys. Rev. D 103, 054504 (2021).

[68] D. Dolgov et al., Nucl. Phys. B Proc. Suppl. 94, 303 (2001).

[69] J.-W. Chen and W. Detmold, Phys. Lett. B 625, 165 (2005).

[70] J.-W. Chen, W. Detmold, J. E. Lynn, and A. Schwenk, Phys. Rev. Lett. 119, 262502 (2017).

[71] J. Lynn, D. Lonardoni, J. Carlson, J. Chen, W. Detmold, S. Gandolfi, and A. Schwenk, J. Phys. G 47, 045109 (2020).

[72] W. Detmold, W. Melnitchouk, J. W. Negele, D. B. Renner, and A. W. Thomas, Phys. Rev. Lett. 87, 172001 (2001).

[73] D. Arndt and M. J. Savage, Nucl. Phys. A697, 429 (2002).

[74] J.-W. Chen and X.-d. Ji, Phys. Lett. B 523, 107 (2001).

[75] A. Parreño, P. E. Shanahan, M. L. Wagman, F. Winter, E. Chang, W. Detmold, and M. Illa, Phys. Rev. D 103, 074511 (2021).

[76] W. Detmold and P. E. Shanahan, Phys. Rev. D 103, 074503 (2021)

[77] T. Uchiyama and K. Saito, Phys. Rev. C 38, 2245 (1988).
[78] I. R. Afnan, F. R. P. Bissey, J. Gomez, A. T. Katramatou, W. Melnitchouk, G. G. Petratos, and A. W. Thomas, Phys. Lett. B 493, 36 (2000).

[79] K. Saito, C. Boros, K. Tsushima, F. R. P. Bissey, I. R. Afnan, and A. W. Thomas, Phys. Lett. B 493, 288 (2000).

[80] W. Bentz, I. C. Cloet, J. T. Londergan, and A. W. Thomas, Phys. Lett. B 693, 462 (2010).

[81] I. C. Cloet, W. Bentz, and A. W. Thomas, Phys. Rev. Lett. 109, 182301 (2012).

[82] A. J. Tropiano, J. J. Ethier, W. Melnitchouk, and N. Sato, Phys. Rev. C 99, 035201 (2019).

[83] B. Schmookler et al. (CLAS Collaboration), Nature (London) 566, 354 (2019).

[84] E. P. Segarra, A. Schmidt, D. W. Higinbotham, E. Piasetzky, M. Strikman, L. B. Weinstein, and O. Hen, Phys. Rev. Lett. 124, 092002 (2020).

[85] I. C. Cloet, W. Bentz, and A. W. Thomas, Phys. Rev. Lett. 102, 252301 (2009).

[86] R. D. Ball, V. Bertone, F. Cerutti, L. Del Debbio, S. Forte, A. Guffanti, N. P. Hartland, J. I. Latorre, J. Rojo, and M. Ubiali, Nucl. Phys. B855, 608 (2012).

[87] J. Mousseau et al. (MINERvA Collaboration), Phys. Rev. D 93, 071101 (2016).

[88] G. G. Petratos et al., Jefferson Lab PAC37 Proposal experiment E12-10-103, 2010.

[89] H.-W. Lin, W. Melnitchouk, A. Prokudin, N. Sato, and H. Shows, Phys. Rev. Lett. 120, 152502 (2018).

[90] R. G. Edwards and B. Joó (SciDAC, LHPC, and UKQCD Collaborations), Nucl. Phys. B Proc. Suppl. 140, 832 (2005).

[91] A. Pochinsky, Qlua, https://usqcd.lns.mit.edu/qlua.

[92] M. Clark, R. Babich, K. Barros, R. Brower, and C. Rebbi, Comput. Phys. Commun. 181, 1517 (2010).

[93] R. Babich, M. A. Clark, and B. Joo, in SC 10 (Supercomputing 2010) (2010), arXiv:1011.0024.

[94] F. Winter, Proc. Sci. LATTICE2013 (2014) 042.

[95] B. Joó, D. D. Kalamkar, T. Kurth, K. Vaidyanathan, and A. Walden, in High Performance Computing, edited by M. Taufer, B. Mohr, and J. M. Kunkel (Springer International Publishing, Cham, 2016), pp. 415-427. 